\title{
A Robust Ensemble Data Method for Identification of Human Joint Mechanical Properties During Movement
}

\author{
Yangming Xu, Member, IEEE, and John M. Hollerbach,* Fellow, IEEE
}

\begin{abstract}
This paper describes a perturbation method for the identification of linear time-varying systems with an unknown input (voluntary joint input) using ensemble data. The method separates the unknown input and the perturbation through highpass filtering and recasts the multi-input single-output system identification into single-input single-output system identification. The method is robust to intertrial variation, and can track changes of system dynamics up to $5 \mathrm{~Hz}$. Analysis and simulation are given for the conditions similar to those for the human arm experiments. Experiments show that mechanical properties of the human elbow joint change with voluntary movement speed and that the mean stiffness with voluntary movement is in the range of the posture and is higher than reported before.
\end{abstract}

Index Terms-Arm movements, ensemble data processing, human joint dynamics, perturbation analysis, system identification.

\section{INTRODUCTION}

$\mathbf{I}$ DENTIFYING the mechanical properties of the human neuromusculoskeleton system under normal conditions is challenging because of the time-varying nature of the system, nonlinearities, unsensed voluntary inputs and adaptation of the central nervous system. Past approaches have relied on apparatuses using electrical or hydraulic actuators that constrain natural human joint movements and limit the results under these conditions. However, the constraint also allows time invariant methods to be used because the human joint is forced to operate under some fixed operating condition. The time invariant methods include frequency methods (transfer function), pulse response function and a sinusoidal method [5]-[7], [10]. The time invariant results obtained are valid only for a fixed operating point. The transient properties of the human joint cannot be obtained with these methods directly.

More recently, a static nonlinear, time-invariant method has been applied to identifying the different contributions to the total joint mechanical properties of the reflex versus intrinsic components [8], [22] by using random position perturbations with hydraulic or electric apparatuses. Again, the approach takes advantage of the constraints of the apparatus on the human joint to obtain tonic contractions.

Manuscript received February 20, 1998; revised September 24, 1998. This work was supported by the Medical Research Council of Canada (MRC) and by NSERC NCE IRIS II Project AMD-2. Asterisk indicates corresponding author.

Y. Xu is with the Sarcos Research Corporation, Salt Lake City, UT 84108 USA.

*J. M. Hollerbach is with the Department of Computer Science, University of Utah, Salt Lake City, UT 84112 USA (e-mail: jmh@cs.utah.edu).

Publisher Item Identifier S 0018-9294(99)02253-3.
To overcome the motion limitations of past apparatuses, we have developed nonrestrictive one-dimensional (1-D) and two-dimensional airjet systems capable of applying random force perturbations to the human joints without any mechanical constraints [15], [16], [21]. The force perturbations can be continuously applied to the human joints without impairing the natural movement. This significant advance in apparatuses brings the possibility of identifying the time-varying human joint mechanical properties under natural movement. Furthermore, unlike position perturbation, small force perturbation does not alter the voluntary action (desired movement).

One of the central issues in studying the mechanical properties of the human arm joints during natural movements using noninvasive methods is to separate the voluntary inputs and movements from the perturbation inputs and perturbed joint movements. Even though EMG's are related to the voluntary actions, they are too noisy to provide reliable information of the voluntary movement. In essence, the voluntary movement is not measurable.

We have developed two methods to separate the voluntary inputs and movements from the perturbation inputs and perturbed joint movements. The first method [17], [19] based on the single trial data applies high-pass filtering to achieve the separation and exponentially weighted least squares estimation method to extract time varying joint mechanical parameters. Due to the limited power in the high-frequency region of the perturbation and the error introduced by high-pass filtering, the method works reasonably well for slow time-varying movements.

The second method described here uses an ensemble method. The ensemble methods are promising [3], [12] because the results at any instance are supported by more data points. The existing methods compute either the impulse response [9], [11]-[13] or the parameters in a model [3]. Early impulse response approaches [9], [11], [13] required manipulating the perturbation input to achieve a strict timing between input and output so that the correlation functions could be computed across the ensemble data. There was a potentially significant error in the final results due to the missed timing. No quantitative error analysis was done. Later, singular value decomposition (SVD) was applied to compute the impulse response directly which relaxed the requirement on the strict timing between input and output [12]. More recently, a model-based approach has been applied [3] with the advantage of producing the physical meaning parameters 
directly, and therefore, giving more direct insight into the motor behavior. The approach presented in this paper belongs to this last category.

All of the previous ensemble data approaches align many trials with similar movement profile based on a feature in the movement profile to calculate an average movement. The perturbed movement is obtained by subtracting the average movement from the movement measurement. Theoretically they can track an instant change of the system dynamics. One of the major problems with the existing ensemble methods is intertrial variation; since the perturbation amplitude is small, the error due to the intertrial variation is often on the same order as the perturbation. Furthermore, the intertrial variation may neither be white nor Gaussian, and may even have nonzero mean. In order to have a stereotyped motion, most of the movements have to be fast. These methods fail for timevarying posture identification because there exists no features at all across different trials, therefore the alignment cannot be done.

In this paper, we propose a robust ensemble data method for time-varying identification of the linearized human arm joint mechanical properties, which is robust to intertrial variation. It has been shown experimentally that under small perturbations, the human joint mechanical properties around a fixed operating point are linear [7]. The time varying approach proposed here allows varying the operating point in real time while the mechanical properties are being identified. It is one experimental step forward toward understanding the movement control. In the following sections, we will first describe the theory for different measurement conditions, then analyze the error and limitations, and finally demonstrate the results by applying it to data from simulated and experimental data sets.

\section{THEORY}

\section{A. Human Forearm Model}

In this paper, we will consider the human forearm dynamics. The equation for the human elbow joint under perturbation can be written as

$$
\ddot{I}=\tau_{t}(\dot{\theta}, \theta)+\tau_{m}(\dot{\theta}, \theta, u)+\tau_{p}+\tau_{g}(\theta)
$$

where $I$ is the moment of inertia of the forearm, $\theta$ is the actual joint angle, $\tau_{t}(\dot{\theta}, \theta)$ is the total torque from the joint passive tissues, $\tau_{m}(\dot{\theta}, \theta, u)$ is the total torque from the muscles, $u$ is the muscle input, $\tau_{p}$ is the perturbation torque and $\tau_{g}$ is the gravity torque. If the two inputs $u$ and $\tau_{p}$ are independent, i.e., $\tau_{p}$ is random, the following linear time-varying model can be derived

$$
I(t) \delta \ddot{\theta}(t)+B(t) \delta \dot{\theta}(t)+K(t) \delta \theta(t)=\tau_{p}(t)
$$

where $I(t), B(t)$, and $K(t)$ are inertia, damping, and stiffness parameters, which reflect the linearized joint mechanical properties at an unperturbed operating point

$$
\delta \theta(t)=\theta(t)-\theta_{n}(t)
$$

is the perturbed joint movement, and $\theta_{n}(t)$ is the joint angle without the perturbation. The goal is to estimate $I(t), B(t)$, and $K(t)$ based on the following input-output measurements: $\tau_{p}$ and $\theta(t)$.

\section{B. Identifying $I(t), B(t)$, and $K(t)$ from Ensemble Data}

The ensemble method requires the collection of a number of input-output realizations, each realization representing the dynamics of the system as it undergoes a similar variation with time. Based on the model (2) and assuming that $\delta \ddot{\theta}, \delta \dot{\theta}$, and $\delta \theta$ have been obtained, an ensemble of $N$ realizations will give rise to a system of $N(>3)$ linear equations, each relating input and output for a particular trial in the ensemble

$$
\tau_{p}(i, t)=I(t) \delta \ddot{\theta}(i, t)+B(t) \delta \dot{\theta}(i, t)+K(t) \delta \theta(i, t)
$$

where $i$ is the realization index. This system of equations may be expressed in matrix form and solved for $\Theta(t)$ using standard least squares

$$
Y(t)=\Phi(t) \Theta(t)
$$

where

$$
\begin{aligned}
Y(t) & =\left\lfloor\tau_{p}(1, t), \cdots \tau_{p}(N, t)\right\rfloor^{T} \\
\Phi(t) & =\left[\begin{array}{c}
\delta \ddot{\theta}(1, t), \delta \dot{\theta}(1, t), \delta \theta(1, t) \\
\vdots \\
\delta \ddot{\theta}(N, t), \delta \dot{\theta}(N, t), \delta \theta(N, t)
\end{array}\right] . \\
\Theta(t) & =\lfloor I(t), B(t), K(t)\rfloor^{T}
\end{aligned}
$$

In the experimental conditions, the angular derivative measurements are not typically available. We avoid direct differentiation by applying a low-pass filter to (4)

$$
\begin{aligned}
L(s)\left[\tau_{p}(i, t)\right]= & L(s)[I(t) \dot{\theta}(i, t)+B(t) \delta \dot{\theta}(i, t) \\
& +K(t) \delta \theta(i, t)]
\end{aligned}
$$

where

$$
L(s)=a^{n} /(s+a)^{n}
$$

$a$ determines the filter cutoff frequency, $n$ is the order of the filter, and $s$ is the Laplace operator. In (6), we have mixed the time and frequency representations for simplifying the explanation. We will occasionally do so when it simplifies the description and the meaning is easily derived from the context. The filter in (7) is well damped to avoid a resonance effect. The operation on the left-hand side is straight forward. We need further manipulation of the right-hand side so that the derivative terms are eliminated. We first examine the first term in the right-hand side of (6)

$$
L(s) I(t) \delta \ddot{\theta}(i, t)=\int_{0}^{t} l(t-\epsilon) I(\epsilon) \ddot{\theta}(i, \epsilon) d \epsilon
$$

where $l(t-\epsilon)$ is the impulse response function of $L(s)$. Since $L(s)$ is a low-pass filter, $l(0)=0$ and $l(t) \approx 0$ when $t>1 / \omega$, we can choose a sufficiently large filter cutoff frequency $\omega$ so that $I(t)$ is approximately constant in the range of $(t-1 / \omega)$ to $t$. Therefore, $I(t)$ can be taken out of the integration. Assume 
$n=2$ in (7), we have

$$
\begin{aligned}
L(s) I(t) \delta \ddot{\theta}(i, t) & \approx I(t) \int_{0}^{t} l(t-\epsilon) \delta \ddot{\theta}(i, \epsilon) d \epsilon \\
& =I(t) \frac{a^{2}}{(s+a)^{2}} s^{2} \delta \theta(i, s) \\
& =I(t)\left[a^{2}-\frac{a^{2}\left(2 a s+a^{2}\right)}{(s+a)^{2}}\right] \delta \theta(i, s) .
\end{aligned}
$$

Again, here we have mixed the time and frequency representations. The second term in the last expression is the proper low-pass filtering on the perturbed angular measurements.

The manipulation on the second term in (6) is simpler, and the third term does not need any manipulation. These manipulations translate (6) into

$$
\begin{aligned}
& \frac{a^{2}}{(s+a)^{2}} s^{2} \tau_{p}(i, s) \\
& \quad=I(t)\left[a^{2}-\frac{a^{2}\left(2 a s+a^{2}\right)}{(s+a)^{2}}\right] \delta \theta(i, s) \\
& \quad+B(t) \frac{a^{2} s}{(s+a)^{2}} \delta \theta(i, s)+K(t) \frac{a^{2}}{(s+a)^{2}} \delta \theta(i, s)
\end{aligned}
$$

where the unknowns are $I(t), B(t)$, and $K(t)$. Now, all of the operations on the $\delta \theta(i, s)$ are proper low-pass filters.

In the experiment, the frequency of the parameter variation is unknown. We may choose an initial filter cutoff frequency based on the joint movement speed and vary it to ensure that the parameter estimates are not significantly affected by the low-pass filter cutoff frequency. Section II-E shows a way to choose the cutoff frequency.

\section{Perturbed Movement and Intertrial Variation}

The accuracy of the above method depends on estimation of the perturbed movements: $\delta \ddot{\theta}(i, t), \delta \dot{\theta}(i, t), \delta \theta(i, t)$ from the angular measurement that includes the voluntary movements. In the experiments, subjects are typically instructed to execute a task in stereotypical fashion and a zero mean random perturbation is applied to the human joint. Some previous methods for estimating $\delta \theta(i, t)$, which we call the ensemble mean removal ensemble data (EMRED) method, follow these steps [3], [12].

1) Lowpass filter each trial:

$$
\theta_{l f}(i, t)={ }^{a} L(s) \theta(i, t)
$$

where ${ }^{a} L(s)$ is an acausal low-pass filter with no phase shift; it can be implemented by using a causal filter, such as:

$$
\frac{s^{n-1} a^{n}}{(s+a)^{n}}
$$

and by passing data forward and then backward once. The purpose to obtain the low-pass filtered position record is to align all of the trials based on common features in the low-pass filtered position record that represent the voluntary movement. This step facilitates the following steps to minimize the variation of the dynamics across the trials in a time slice. The lowpass filter cutoff frequency is equal to the movement frequency.

2) Align all trials based on a feature in the low-pass filtered data $\theta_{l f}(i, t)$, such as peak velocity, midpoint between two target points, or minimum area. For our simulation and experiments, we used the minimum area of the difference between the average position record from all of the low-pass filtered position records and each individual position record.

3) Compute the ensemble averages $\bar{\theta}(t)$ and $\bar{\tau}(t)$ of all trials and standard deviations $\sigma_{\theta}(t)$ at each time step $t$

$$
\begin{aligned}
\bar{\theta}(t) & =\frac{1}{N} \sum_{i}^{N} \theta(i, t) \\
\bar{\tau}(t) & =\frac{1}{N} \sum_{i}^{N} \tau(i, t) \\
\sigma_{\theta}(t) & =\sqrt{\frac{1}{N-1} \sum_{i}^{N}[\theta(i, t)-\bar{\theta}(t)]^{2}} .
\end{aligned}
$$

4) Reject trials whose low-pass filtered records deviate from the ensemble average by more than one standard deviation, i.e., for any $t$, if

$$
\left|\bar{\theta}(t)-\theta_{l f}(i, t)\right|>\left|\sigma_{\theta}(t)\right|
$$

the trial is thrown out.

5) Compute the responses due to perturbation for the identification by

$$
\begin{aligned}
& \delta \theta(i, t)=\theta(i, t)-\bar{\theta}(t) \\
& \delta \tau(i, t)=\tau(i, t)-\bar{\tau}(t) .
\end{aligned}
$$

6) Use the method outlined in Section II-B to identify the parameters.

Step 4 implies that the error due to intertrial variation can be up to one standard deviation, which can be on the same order as the perturbation amplitude. This amount of intertrial variation makes the stiffness artificially low.

\section{High-Pass Mean Removal Ensemble Data (HMRED) Method}

We suggest to solve the problem of intertrial variation, by separating the voluntary movement and perturbed movement in frequency, and extracting the perturbed movements from each experimental trial by high-pass filtering. The procedure is as follows:

Steps 1-4 are the same as those in EMRED.

5) High-pass filter each trial

$$
\begin{aligned}
& \delta \tilde{\theta}(i, t)={ }^{q} H(s) \theta(i, t) \\
& \delta \tilde{\tau}(i, t)={ }^{q} H(s) \tau(i, t)
\end{aligned}
$$

where

$$
{ }^{q} H(s)=1-{ }^{a} L(s)
$$

is an acausal high-pass filter, $\theta(i, t)$ is the joint angle measurement given by (3). 


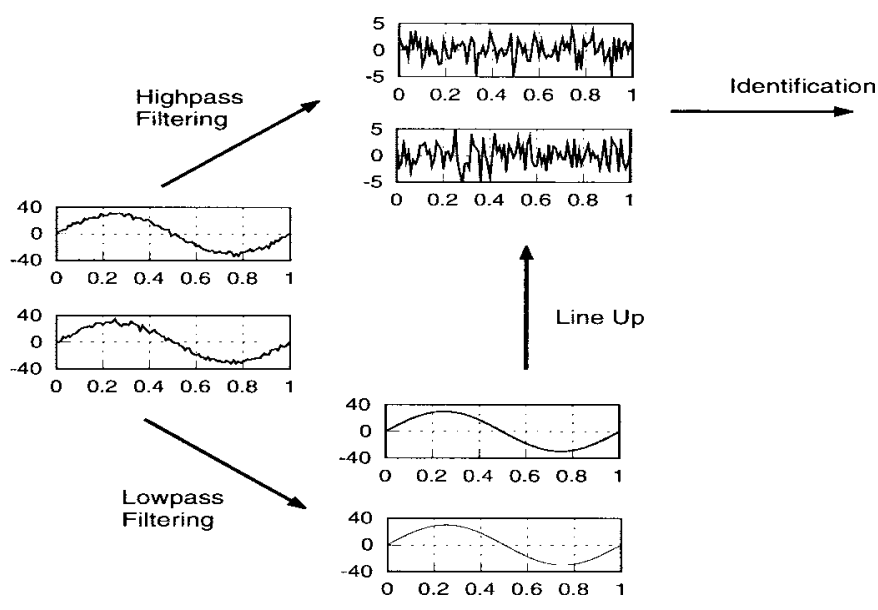

Fig. 1. Block diagram of the identification procedure of the HMRED method.

6) Align high-pass filtered position and torque data according to the recorded amount of shift in time in Step 2 using the low-pass filtered data. Fig. 1 shows the above procedure graphically.

7) Use the method outlined in Section II-B to identify the parameters.

\section{E. Effect of High-Pass Filtering}

The effect of high-pass filtering is analyzed by examining if the following expression is approximately equal to zero and using (3) and (16)-(18) for the derivations

$$
\begin{aligned}
& \delta[I(t) \delta \ddot{\tilde{\theta}}(i, t)+B(t) \delta \dot{\tilde{\theta}}(i, t)+K(t) \delta \tilde{\theta}(i, t)]-\tilde{\tau}(i, t) \\
&= I(t)\left[\ddot{\theta}_{n}(i, t)+\delta \ddot{\theta}(i, t)-{ }^{a} L(s) \ddot{\theta}_{n}(i, t)\right. \\
&\left.-{ }^{a} L(s) \delta \ddot{\theta}(i, t)\right]+B(t)\left[\dot{\theta}_{n}(i, t)+\delta \dot{\theta}(i, t)\right. \\
&\left.-{ }^{a} L(s) \dot{\theta}_{n}(i, t)-{ }^{a} L(s) \delta \dot{\theta}(i, t)\right] \\
&+K(t)\left[\theta_{n}(i, t)+\delta \theta(i, t)-{ }^{a} L(s) \theta_{n}(i, t)\right. \\
&\left.-{ }^{a} L(s) \delta \theta(i, t)\right]-\left[\tau(i, t)-{ }^{a} L(s) \tau(i, t)\right] \\
&= I(t)\left[\ddot{\theta}_{n}(i, t)-{ }^{a} L(s) \ddot{\theta}_{n}(i, t)\right]+B(t)\left[\dot{\theta}_{n}(i, t)\right. \\
&\left.-{ }^{a} L(s) \dot{\theta}_{n}(i, t)\right]+K(t)\left[\theta_{n}(i, t)\right. \\
&\left.-{ }^{a} L(s) \theta_{n}(i, t)\right]+[I(t) \delta \ddot{\theta}(i, t) \\
&+B(t) \delta \dot{\theta}(i, t)+K(t) \delta \theta(i, t)-\tau(i, t)] \\
&-[I(t) \mathcal{L}(s) \delta \ddot{\theta}(i, t)+B(t) \mathcal{L}(s) \delta \dot{\theta}(i, t) \\
&\left.+K(t) \mathcal{L}(s) \delta \theta(i, t)-{ }^{a} L(s) \tau(i, t)\right] \\
& \approx 0+0+0+0 \\
&-{ }^{a} L(s)[I(t) \delta \ddot{\theta}(i, t)+B(t) \delta \dot{\theta}(i, t) \\
&+K(t) \delta \theta(i, t)-\tau(i, t)] \\
&= 0 .
\end{aligned}
$$

In the second step, the first three terms are equal to zero individually because the voluntary movements are low-frequency. The fourth term is equal to zero because of (2). The last step uses the same assumption as Section II-B that the parameters $I(t), B(t)$ and $K(t)$ are approximately constant in the range of the filter response time.

Using the low-pass filter to facilitate the identification without the derivative variables in Section II-B and the high-pass filter to remove the voluntary movement in the measurements implies applying the bandpass filter to the measurements. High-pass filter (18) translates a high-pass operation into lowpass operation. An acausal low-pass filter is preferred to avoid phase shift. The same type of the low-pass filter but different cutoff frequency for the low-pass and high-pass filters is used in the simulation and experiments. It is apparent that the lowpass filter cutoff frequency in Section II-B must be higher than the high-pass filter cutoff frequency or the low-pass filter cutoff frequency in (18) in order to have sufficient signal power left over in the processed data. Furthermore, if the low-pass filter cutoff frequency in (19) is sufficiently high to have the approximate equality valid (19), the low-pass filter cutoff frequency in Section II-B would be sufficient. For the simulation and experiments, the low-pass filter cutoff frequency is $10 \mathrm{~Hz}$ higher than the high-pass filter cutoff frequency even though simulation tests show that sometimes $5 \mathrm{~Hz}$ is sufficient to give the same result in terms of the variation of the parameter estimates versus the frequency. The higher the low-pass filter cutoff frequency the stronger the effect of the high-frequency noise. The $10-\mathrm{Hz}$ low-pass filter cutoff frequency is determined based on the simulation results using the similar conditions in our experiments. The high-pass filter cutoff frequency is determined experimentally in both simulation and experiments.

The analysis does not indicate any requirement on the filter type. We have tried different filter types (Butterworth and Chebyshev) to both simulation and experimental data, and found that the filter type does not affect the parameter estimates as long as the filter is well or critically dampened. If the filter is under-dampened, the parameter estimates become noisy. In our simulation and experiments, the Butterworth filter is used.

\section{SimUlation}

In this section, we examine the performance of the EMRED, HMRED, and exponentially weighted least squares (EWLS) methods [20] in a set of simulated conditions similar to those in the experiments. EWLS is a single trial method and therefore has no intertrial variation problem. We will compare its performance and experimental results in the next section to those of EMRED and HMRED. We would also like to find the application boundary for the HMRED method for experimental design.

For the EMRED and HMRED methods, each uses about 400 trials. Under noise free condition, three trials are sufficient to identify the parameters. More trials improve the sensitivity of both methods to the high-frequency noise. However, over 200 trials, the rate of the improvement becomes slow for the given maximum noise level used in the simulation.

The simulation model is similar to (2) with addition of the voluntary input $\tau_{v}(t)$

$$
I(t) \delta \ddot{\theta}(t)+B(t) \delta \dot{\theta}(t)+K(t) \delta \theta(t)=\tau_{v}(t)+\tau_{p}(t) .
$$

In the simulation, $I(t)$ is assumed to be constant; $B(t)$ and $K(t)$ are assumed to be sinusoidally time-varying with the same frequency. For example, for $K(t)$

$$
K(t)=K_{m v}+K_{v a} \sin (2 \pi f t)
$$




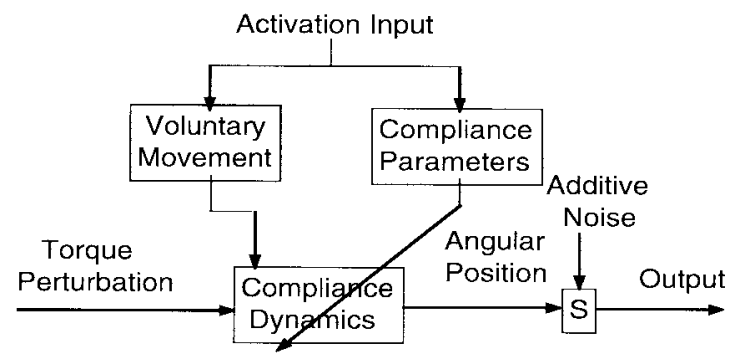

Fig. 2. Block diagram of the simulation procedure for identification of the time-varying elbow joint dynamics in posture and movement.

TABLE I

Nominal Model Parameters USEd in the Simulation

\begin{tabular}{c|c|c}
\hline Variable & Mean Value & Variation Amplitude \\
\hline$I\left(\mathrm{Nms}^{-2} / \mathrm{rad}\right)$ & 0.06 & 0 \\
$B\left(\mathrm{Nms}^{-1} / \mathrm{rad}\right)$ & 1.8 & 1 \\
$K(\mathrm{Nm} / \mathrm{rad})$ & 10. & 15 \\
\hline
\end{tabular}

where $K_{m v}$ is the mean value, $K_{v a}$ is the variation amplitude, and $f$ is the frequency. The damping ratio is kept constant at 0.6 . The mean and variation of the parameters are given in Table I. The voluntary movement is assumed to have a sinusoidal equilibrium trajectory

$$
\tau_{v}(t)=K(t) \sin (2 \pi f t) .
$$

The simulation procedure is shown in Fig. 2. Pseudorandom binary perturbation prefiltered by a low-pass filter with a cutoff frequency at $150 \mathrm{~Hz}$, which mimics the D/A converter, is used for $\tau_{p}$. The root-mean-square value of the perturbation is $4 \mathrm{Nm}$, which is less than $10 \%$ of the voluntary input. The joint angle measurement is $\theta(t)$ given in (3). We assume 5\% noise on the torque measurement and $1 \%$ noise on the angular position measurement in terms of the standard deviation of the amplitude. These values are consistent with the accuracy of our airjet system described in Section IV-A.

For the HMRED method, the high-pass filter cutoff frequency for (16) and (17) is $5 \mathrm{~Hz}$, determined from Fig. 5; the low-pass filter cutoff frequency for (6) is $15 \mathrm{~Hz}$; and the lowpass filter cutoff frequency for the step one in Section II-C is equal to the voluntary movement frequency (22). The filter type is Butterworth.

We evaluate the estimation by using variance-accounted-for of the mean (VAFM) and of the variance (VAFV) of output prediction and parameter estimates, which are defined as

$$
\begin{aligned}
& \mathrm{VAFM}=\left[1-\frac{(\overline{y(t)}-\overline{\hat{y}(t)})^{2}}{\overline{y(t)}^{2}}\right] \cdot 100 \% \\
& \mathrm{VAFV}=\left[1-\int_{0}^{t} \frac{(\tilde{y}(t)-\tilde{\hat{y}}(t))^{2}}{\tilde{y}^{2}(t)} d t\right] \cdot 100 \%
\end{aligned}
$$

where $\overline{y(t)}$ is the mean of $y(t)$ over $t, \tilde{y}(t)$ is $(y(t)-\overline{y(t)})$, and $\tilde{\hat{y}}(t)$ is $(\hat{y}(t)-\overline{\hat{y}(t)})$.

Both VAFM and VAFV will be meaningless if their denominators are close to zero. For example, the inertia in the following simulation is assumed to be constant, $\tilde{y}(t)=0$. We

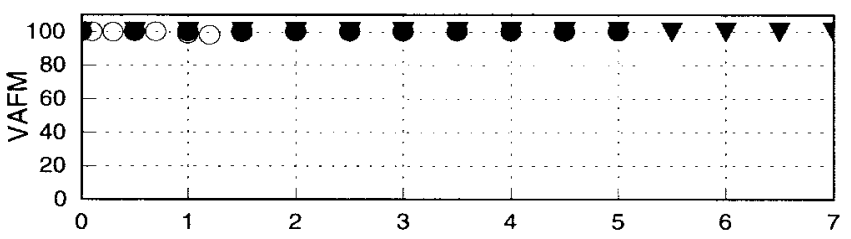

(a)

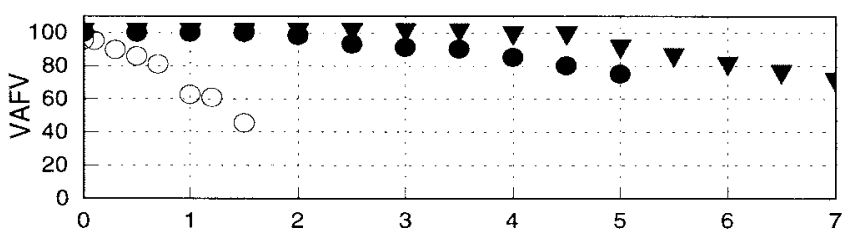

(b)

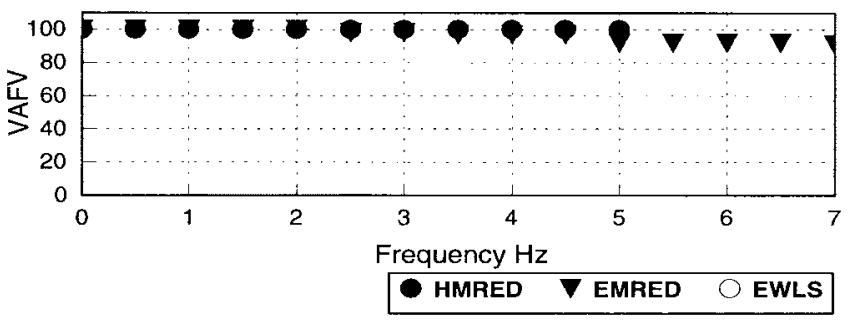

(c)

Fig. 3. Simulation: VAFV of HMRED, EMRED, and EWLS versus the frequency of the parameter variation with no intertrial variation.

suggest that when the denominator is less than one, we set it to one. When a signal is zero mean, (24) is the same as the VAF definition in [12]. We require both VAFM and VAFV over $75 \%$ [3], [12], for which the relative prediction error variance is less than one third.

Fig. 3 compares the frequency responses of VAFV of the estimated stiffness versus parameter variation frequency without any intertrial variations for HMRED, EMRED and EWLS. The high-pass filter cutoff frequency in HMRED is equal to the movement frequency plus two. The VAFV of the stiffness estimate for HMRED decreases below $75 \%$ after $5 \mathrm{~Hz}$, while that for EMRED is below $70 \%$ after $7 \mathrm{~Hz}$. Compared to EWLS whose VAFV is below $70 \%$ after $0.5 \mathrm{~Hz}$, HMRED improves the frequency of the parameter tracking performance by ten times. The VAFV's of the inertia and damping estimates and the VAFM's of all three parameters (not shown) also vary with the frequency of the parameter variation, but much less than that of the stiffness estimate. In the tested frequency range the VAFV of the output prediction is always above $95 \%$ for all methods.

Fig. 4 shows the effect of intertrial variation to the stiffness estimation and output prediction for HMRED and EMRED. The other parameters are affected less, and are not shown here. In the simulation the voluntary movement frequency is perturbed by a zero mean, normal distribution intertrial variation. The standard deviation is the measure of the strength of intertrial variation. The voluntary movement frequency is 2 $\mathrm{Hz}$ and the cutoff frequency of the high-pass filter for HMRED is $5 \mathrm{~Hz}$.

- The VAFM of the stiffness estimate of EMRED decreases below $70 \%$ after the standard deviation of the intertrial 


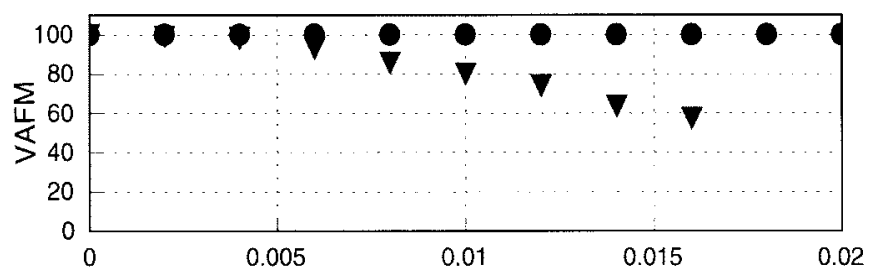

(a)

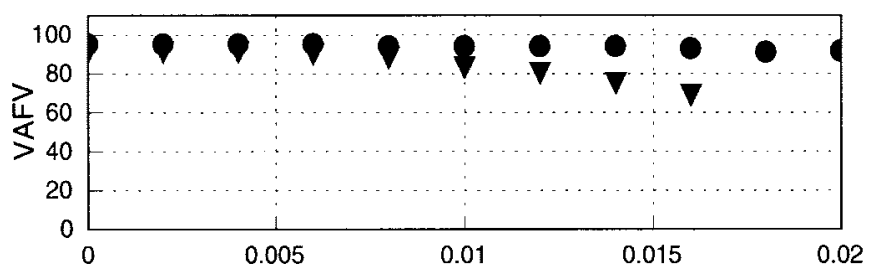

(b)

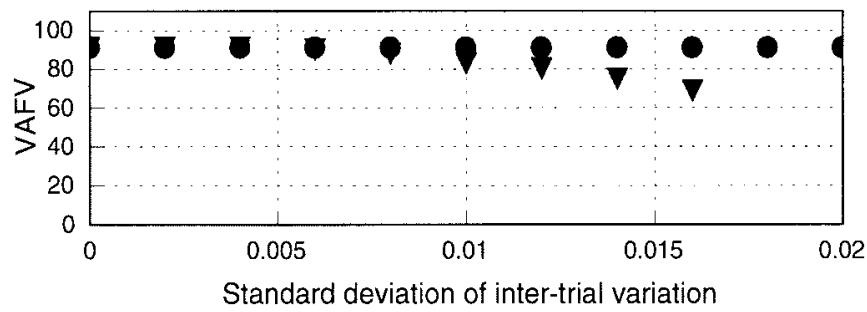

$\checkmark$ EMREM HMREM

(c)

Fig. 4. Simulation: VAFM and VAFV of the stiffness estimates, and VAFV of the output prediction of EMRED and HMRED versus standard deviation of intertrial variation.

variation (SDITV) is over 0.013 while that of HMRED stays at $100 \%$. Even at 0.02 of the SDITV, the VAFM of the stiffness estimate from HMRED is still close to $100 \%$, which indicates the robustness of HMRED to the intertrial variation.

- The VAFV's of the stiffness estimates and output prediction of both EMRED and HMRED decrease with intertrial variation. However the VAFV of the HMRED stiffness decreases slower than that of EMRED.

- Even though not shown here, the VAFM of the damping estimate of EMRED also decreases with the SDITV while that of HMRED stays close to $100 \%$. The VAFM and VAFV of the inertia estimate for both EMRED and HMRED do not vary with the SDITV, and are close to $100 \%$.

Fig. 5 shows the VAFM and VAFV of the parameter estimates from the HMRED versus the high-pass filter cutoff frequency. In the simulation the parameter variation frequency is $2 \mathrm{~Hz}$ and the standard deviation of intertrial variation is 0.01 . The VAFM and VAFV of the damping and stiffness estimates as well as the output prediction show that between 4 and $6 \mathrm{~Hz}$, the VAFM and VAFV are above $95 \%$. Over $6 \mathrm{~Hz}$, both VAFM of the damping estimate and VAFV of the stiffness estimate start decreasing. The inertia is not affected.

From the simulation we can conclude that under the conditions: 1) no derivative measurements, 2) the output noise is less than $5 \%$ and input noise less than $1 \%$, and 3) small

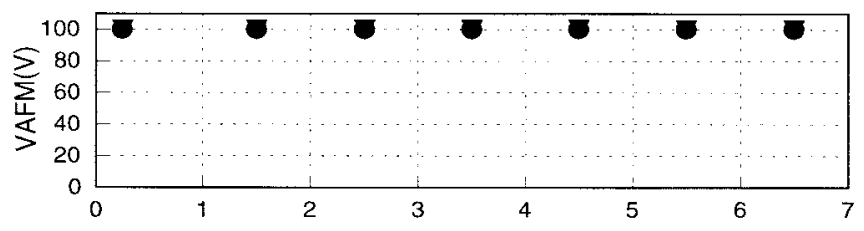

(a)

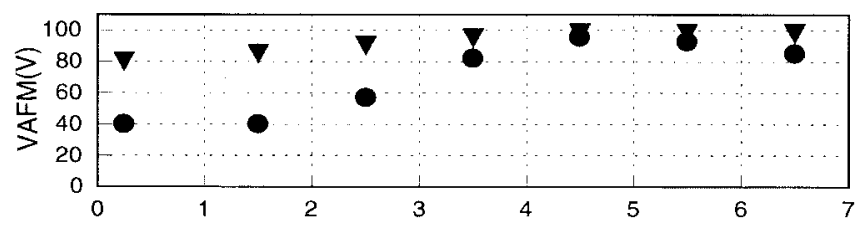

(b)

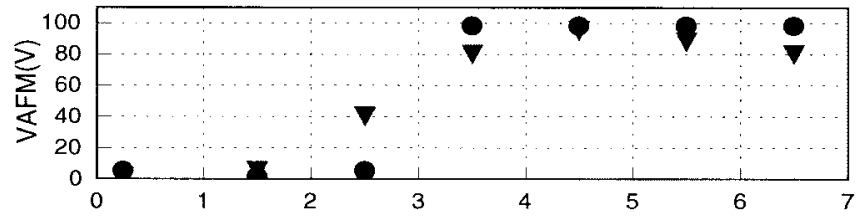

(c)

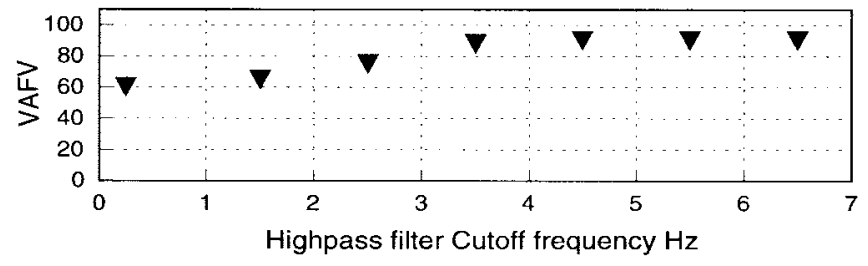

VAFM VAFV

(d)

Fig. 5. Simulation: VAFM and VAFV of HMRED versus the high-pass filter cutoff frequency.

force perturbation, less than $10 \%$ of the voluntary input, the HMRED method can track the parameter variation up to $5 \mathrm{~Hz}$. It is less sensitive to intertrial variation than EMRED.

\section{EXPERIMENTAL METHODS}

The results of the simulation guide the experimental design for study of the human elbow joint dynamics during movement.

\section{A. Apparatus}

The airjet system [21] consists of three parts: an airjet actuator, a motion tracking device (Optotrak, Northern Digital Inc., Waterloo, Ont, Canada), and a VME Bus-based data acquisition system. Fig. 6 shows how the airjet actuator is attached to a subject's wrist. The airjet force is sensed by a strain gauge force sensor which has noise less than $5 \%$ [21]. The airjet actuator is currently a 1-D perturbation device, capable of producing $\pm 4 N$ forces from the reaction force of compressed air and of switching the force up to $80 \mathrm{~Hz}$, based on the Coanda effect. In the experiment the airjet actuator was controlled to produce a pseudorandom binary sequence (PRBS) through a digital-to-analog converter (DAC) channel at a $100-\mathrm{Hz}$ sampling frequency. The force signal was col- 


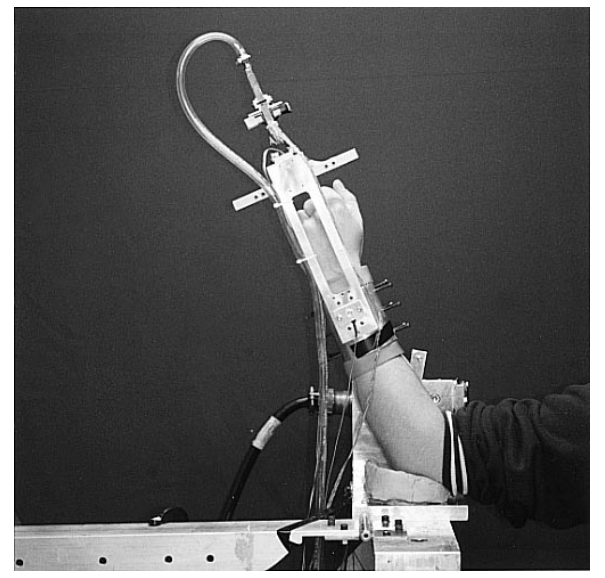

Fig. 6. The airjet actuator attached to a subject's wrist.

lected at $1 \mathrm{kHz}$. The Optotrak uses three LCD cameras to track infrared light-emitting diodes (IRED) and produces threedimensional position data of the IRED's with an accuracy of $0.1 \mathrm{~mm}$. One IRED was used in the experiments and glued to the subject's wrist area. The Optotrak was sampled at 0.5 $\mathrm{kHz}$ and resampled digitally to $1 \mathrm{kHz}$ later to match the force sampling frequency.

\section{B. Subjects}

Four right-handed subjects (one of them is female) with no history of neuromuscular disease, ranging in age from 22 to $32 \mathrm{yr}$, were examined. The subject sat in a chair and rested the left elbow on a piece of clay on the bench. The elbow length was estimated by using both caliber measurement and the Optotrak; results agreed within $2 \%$. The forearm was free to move in a vertical plane passing through the upper arm. The subject's palm was turned $90^{\circ}$ away from his shoulder. The wrist was immobilized through a custom fitted plastic cuff on which the airjet actuator is strapped.

A physical moving target is presented to the subject at the wrist area. The target moves around a center point aligned with the elbow joint center. The trajectory of the target is sinusoidal, $\pm 28^{\circ}$ about the vertical line. Three different frequencies were used: $0.25,0.5$, and $1 \mathrm{~Hz}$. The target does not touch the subject's wrist. The task was not difficult for the subject when the cyclic movement is less than $2 \mathrm{~Hz}$. When the target motion is $2 \mathrm{~Hz}$ or more, some subjects could not follow the target. Subjects were instructed to use the effort that they felt comfortable with throughout the experiments that typically took about $1.5 \mathrm{~h}$.

Each frequency is repeated 15 times. There is 1-2 min rest period between each repetition. Between two difference frequencies, there is 4-min rest period.

\section{Data Analysis}

As in the simulation, for the HMRED method, the highpass filter cutoff frequency is determined by examining the parameter estimate versus the cutoff frequency (see below). The low-pass filter for (6) is $10 \mathrm{~Hz}$ higher than the highpass filter cutoff frequency. Varying the low-pass filter cutoff frequency by $50 \%$ (maximum) changed the parameter estimate

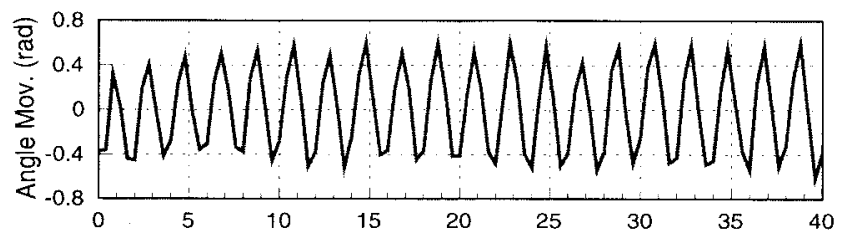

(a)

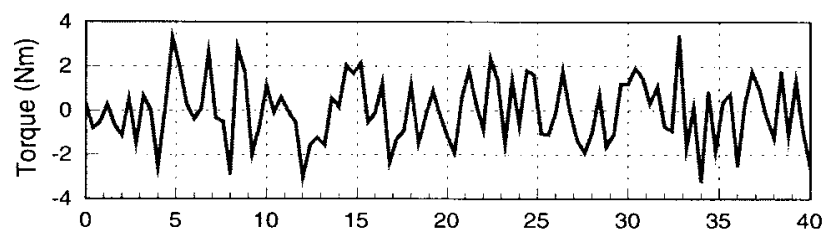

(b)

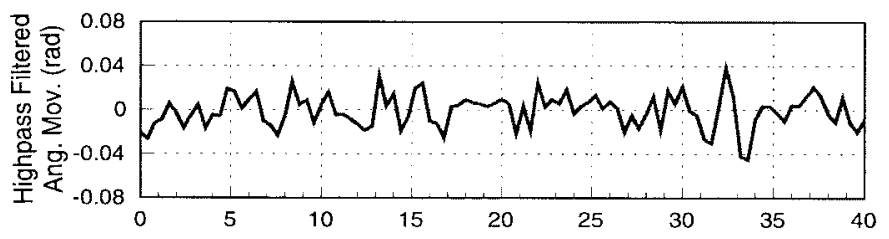

(c)

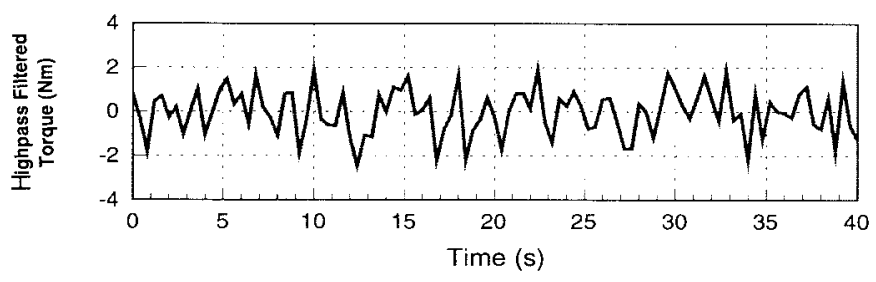

(d)

Fig. 7. Experiment: (a) joint angle, (b) perturbation torque, (c) high-pass filtered joint angle, and (d) high-pass filtered perturbation torque records from a $0.5-\mathrm{Hz}$ experiment (Subject PZ)

by less than 5\% VAF. The low-pass filter cutoff frequency for step one in Section II-C is equal to the voluntary movement frequency.

Fig. 7(a) and (b) shows typical force and position records from one experiment $(0.5-\mathrm{Hz}$ voluntary movement). We define one trial in one experiment as a complete cyclic movement. In Fig. 7 there are 45 trials (only 20 trials are shown for presentation clarity). Fig. 7(c) and (d) shows the high-pass filtered data from the same experiment. The cutoff frequency was $2 \mathrm{~Hz}$. The low-frequency voluntary movement is clearly removed from the position record. The perturbed joint movement is about $5 \%$ of the voluntary movements. Fig. 8 shows the ensemble-aligned joint angle data, based on the method outlined in Section II-D. The variation across trials is due to the perturbation and intertrial variation, but the aligned trials are very similar. To reduce the noise effect we used at least 400 trials from several experiments for each case.

The cutoff frequency of the high-pass filter in HMRED must be chosen properly in order to obtain accurate results. In the simulation, at certain values of the high-pass filter cutoff frequency, there is a clear corner in the plot of the mean and standard deviation of the stiffness versus the cutoff frequency (Fig. 5). With the experimental data, we must determine a suitable high-pass cutoff frequency for each 


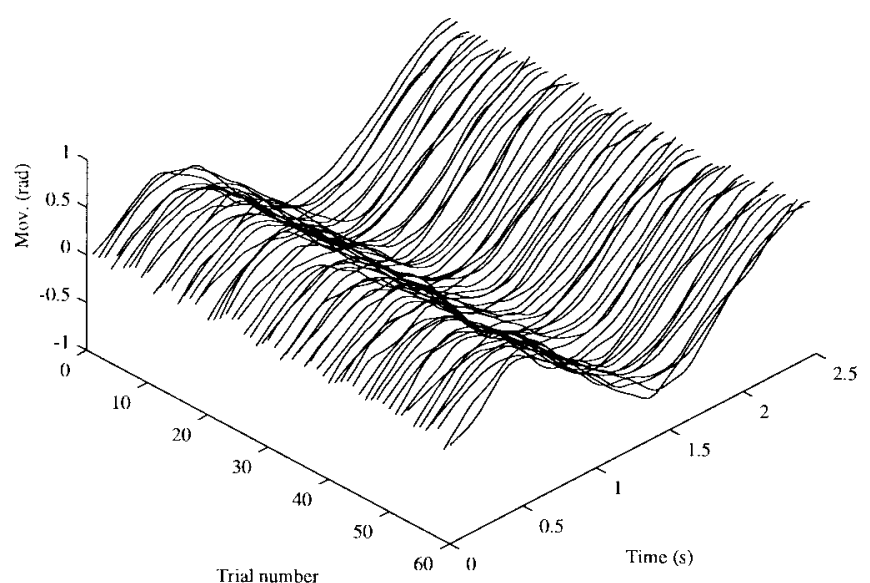

Fig. 8. Experiment: waterfall plots of the aligned angle movements from a 0.5-Hz experiment (Subject PZ).

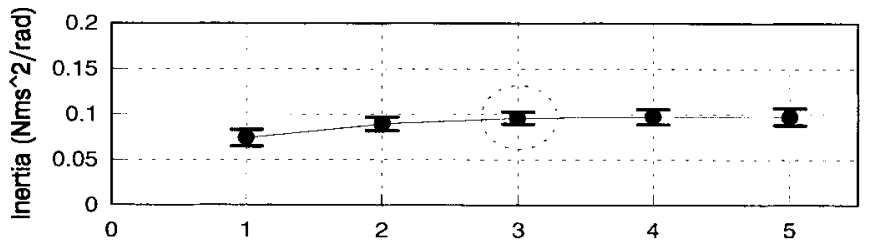

(a)

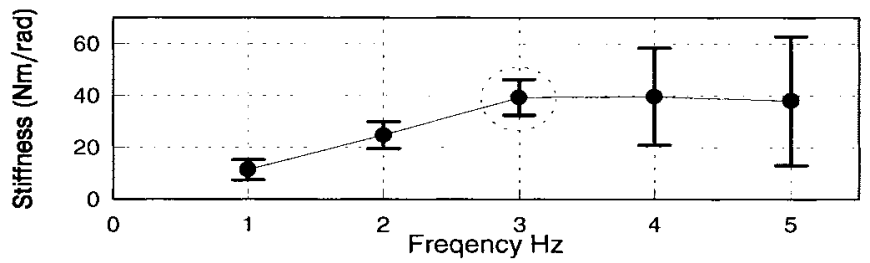

(b)

Fig. 9. Experiment: mean and standard deviation of the estimated inertia and stiffness versus the high-pass filter cutoff frequency (Subject DHA). The standard deviation is calculated after the data has been high-pass filtered at the voluntary movement frequency.

voluntary movement frequency. Fig. 9 shows the mean and standard deviation of the inertia and stiffness versus the high-pass filter cutoff frequency for the $0.5-\mathrm{Hz}$ voluntary movements. Below $3 \mathrm{~Hz}$, the mean values of the estimated inertia and stiffness increase with the high-pass filter cutoff frequency, which must be due to the effect of the intertrial variation. The mean values have the corner frequency at $3 \mathrm{~Hz}$. From 3 to $4 \mathrm{~Hz}$ there is a large increase of the standard deviation of the stiffness. Therefore, $3 \mathrm{~Hz}$ should be the highpass filter cutoff frequency. We made one plot similar to Fig. 9 for each voluntary movement for each subject to determine the cutoff frequency accordingly.

\section{EXPERIMENTAL RESULTS}

We tested three movement speeds: $0.25,0.5 \mathrm{~Hz}$, and $1 \mathrm{~Hz}$. The results are summarized below.

\section{A. $0.25-\mathrm{Hz}$ Voluntary Movement}

Fig. 10 shows typical results of the stiffness estimate, VAFV and voluntary movement trajectory from the 0.25 voluntary

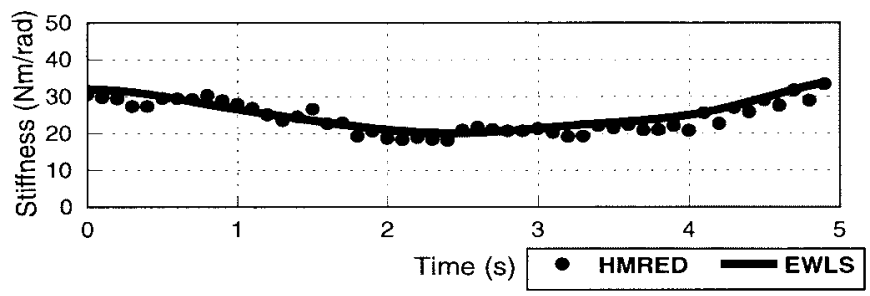

(a)

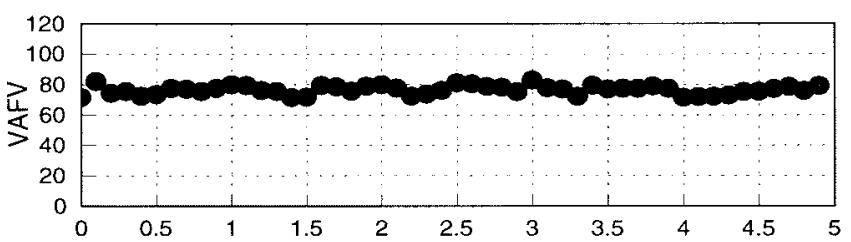

(b)

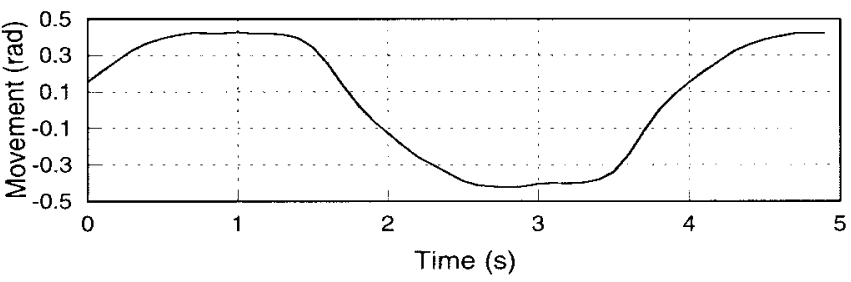

(c)

Fig. 10. Experiment: comparison of the EWLS and HMRED methods (Subject DHA).

movement experiment. At such a low-frequency, EWLS works as well [19], and its results are shown to agree well with HMRED. Furthermore, both methods have the VAFV of the output prediction over $75 \%$.

The mean value of the estimated inertia, damping, stiffness and damping ratio are similar to those from the postural experiments [19] (only the stiffness is shown in Fig. 10). The estimated inertia does not vary which is expected. The mean value of the damping ratio is low: 0.3 , and the stiffness shows a low-frequency variation with a mean of $25 \mathrm{Nm} / \mathrm{rad}$ which is close to or slightly higher than that from the minimum effort posture [19]. By visual inspection, there is no clear correlation between the stiffness and movement variations. The computed cross correlation coefficient is below 0.4 .

\section{B. $0.5-\mathrm{Hz}$ Voluntary Movement}

Fig. 11 shows the results from the $0.5-\mathrm{Hz}$ voluntary movement experiments. The VAFV of the output prediction is approximately $80 \%$. Fig. 11 shows the data from two subjects.

- The inertia for each is approximately constant with time, but differs across subjects (not shown).

- The stiffness and damping vary with time, do not vary consistently across subjects, and show no correlation with the voluntary movement (the computed correlation coefficients are below 0.5). The mean value of the stiffness is similar to those from our previous experiments with the minimum effort posture while the mean value of the damping is similar to those from our previous experiments with the medium effort posture [19]. 


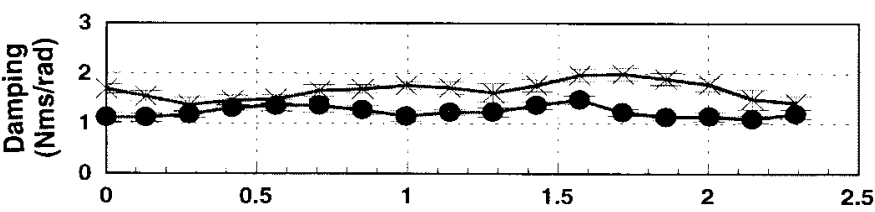

(a)

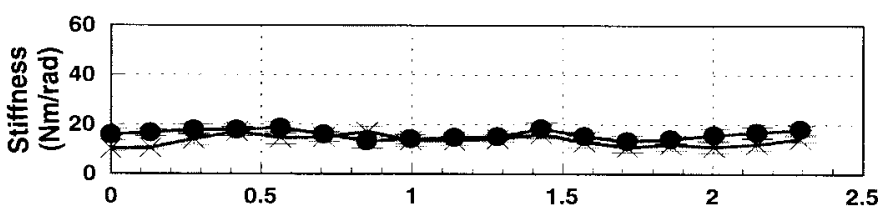

(b)

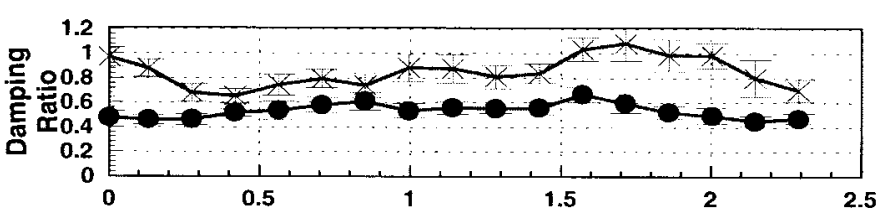

(c)

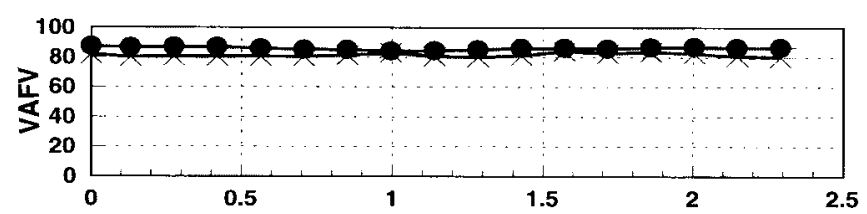

(d)

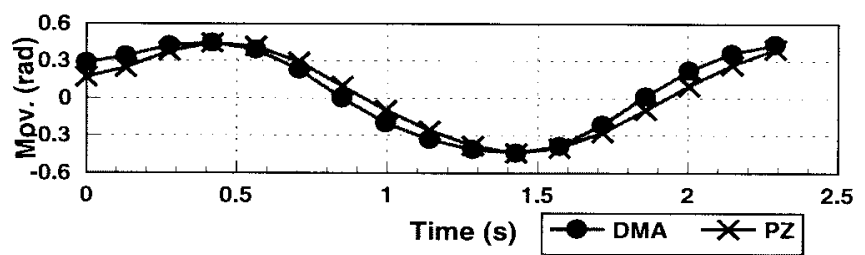

(e)

Fig. 11. Experiment: results from two subjects for $0.5-\mathrm{Hz}$ voluntary movement.

- The mean values of the damping ratio for subjects PZ and YXU are around 0.7. In general the mean values for all subjects are higher than those from our previous experiments [19]. However, the damping ratios have considerable variation (Fig. 11).

\section{C. $1-\mathrm{Hz}$ Voluntary Movement}

Fig. 12 shows the results from the $1-\mathrm{Hz}$ voluntary movement experiments. The VAFV of the output prediction is above $75 \%$.

- Similar to the experiments with $0.5-\mathrm{Hz}$ voluntary movement (Fig. 11), the inertia is constant and differs across subjects. The mean value for each subject and the amount of the difference across subjects are similar to those from the experiments with $0.5-\mathrm{Hz}$ voluntary movement.

- The stiffness, damping and damping ratio vary with time and have a clear correlation with the voluntary movement. The stiffness is higher at the two target points. The mean value of the stiffness is doubled compared to that from the $0.5-\mathrm{Hz}$ voluntary movement experiments, and is

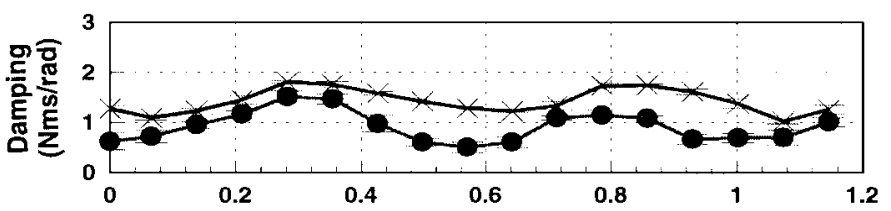

(a)

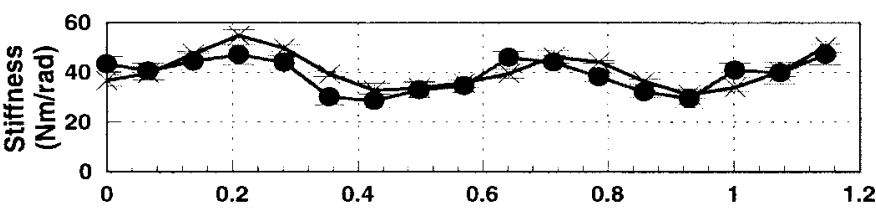

(b)

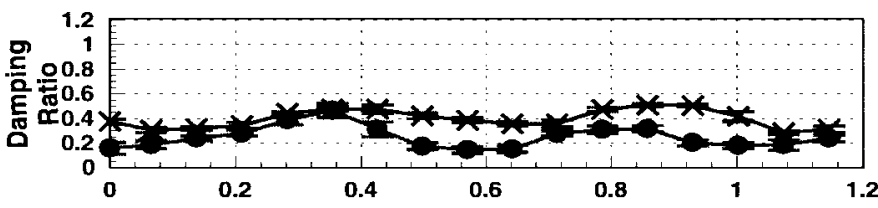

(c)

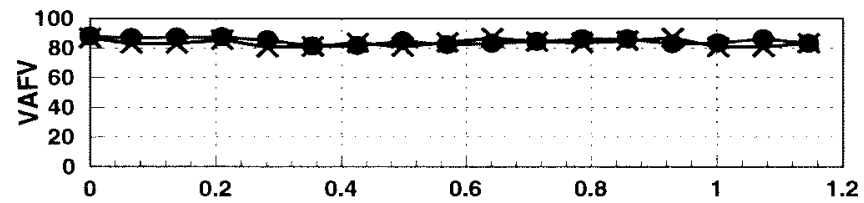

(d)

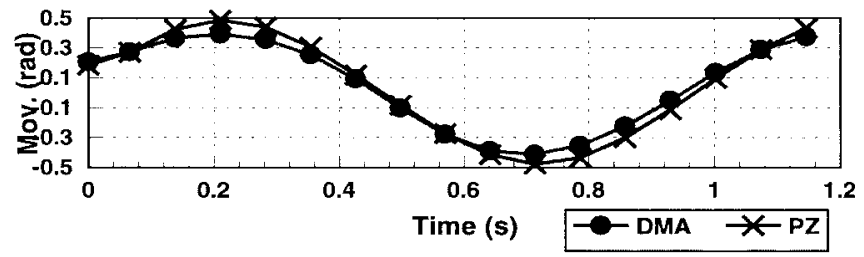

(e)

Fig. 12. Experiment: results from 1-Hz voluntary movement: (a) inertia, (b) damping, and (c) stiffness.

two-thirds of that from the $0.25-\mathrm{Hz}$ voluntary movement experiments.

- The damping shows a pattern similar to the stiffness, except that there is about a $0.1 \mathrm{~s}$ time lag. The mean value of the damping is smaller than that in the previous experiments with 0.25 and $0.5-\mathrm{Hz}$ voluntary movement. The damping ratio has a pattern similar to the damping, and the mean value is around 0.3 which is similar to that obtained in the postural experiments [19].

Except for the mean value, the time-varying pattern of the stiffness and damping is consistent with that reported in [3].

\section{DISCUSSION}

This paper demonstrates that the HMRED method is a successful means to identify time-varying dynamics of the human motor control system. This method has the following four major advantages:

- it is robust to intertrial variation;

- it can handle reasonably fast time-varying dynamics; 
- few restrictions are placed on the input signal;

- the error due to the method itself can be characterized.

Under our experimental conditions (small force perturbation, no derivative measurements, linear or linearized systems, and existence of the unknown voluntary actions), the technique estimates the time-varying parameters up to a frequency of $5 \mathrm{~Hz}$. The method is robust to output noise, has been tested extensively, and its limitations have been explored.

In identifying time-varying dynamics in movement, we have shown that previous ensemble-based methods are sensitive to intertrial variation. We showed the effect of intertrial variation is worse than that of the measurement noise. The major effect of intertrial variation is to lower the stiffness mean value.

The primary objective of the experimental study was to establish the viability of the HMRED method. Nevertheless, the results are of considerable physiological interest.

- The time-invariant inertia estimate for each subject validates our linearization assumption.

- The mean values of stiffness, damping and damping ratio decrease from $0.25-0.5 \mathrm{~Hz}$, then increase from $0.5-1 \mathrm{~Hz}$. Considering the results from posture and slow movements $(0.125 \mathrm{~Hz})$ in our previous study using the EWLS method [19], the stiffness of the elbow joint seems to decrease from the posture to slow movement and then to increase.

- With $1-\mathrm{Hz}$ movement, there is a consistent pattern of the stiffness and damping variation across subjects. The stiffness is higher at the target points, which is consistent with [3]. At lower frequencies no patterns can be seen.

- The stiffness mean values from our movement experiments are within the range of the stiffness mean values from the minimum and maximum effort posture experiments [19].

- The damping ratio from $0.5-\mathrm{Hz}$ movement is considerably higher than that reported before from posture and movement under different conditions. This is the first time that we observe that the damping ratio reaches the critically damped point.

Except for the mean values, our findings are largely consistent with and extend those reported before. Lanman [10] reported a decrease in the stiffness with speed of movement based on fixed sinusoidal perturbation tests. Judging from his figures, the maximum speed was $10 \%$ or $0.174 \mathrm{rad} / \mathrm{s}$. The voluntary movement frequency, maximum speed, and the mean stiffness from our movement experiments (Figs. 10-12) with a movement amplitude of $0.5 \mathrm{rad}$ are listed in Table II. Our lowest speed is higher than his highest one. We actually demonstrate that the stiffness decreases further with speed.

At the $1-\mathrm{Hz}$ voluntary movement, the time-varying patterns of the stiffness, damping and damping ratio are consistent with those reported by Bennett et al. [3]. The same airjet was used in their experiments, and the voluntary movements were cyclic at 0.5 and $0.67 \mathrm{~Hz}$. They found that 1) the stiffness was higher at the target limit points and 2) the mean of the stiffness increases with the movement speed. In our experiments we observe that the stiffness is higher at the target points only at the $1-\mathrm{Hz}$ voluntary movements, not at the $0.5-\mathrm{Hz}$ voluntary movements, and the stiffness increases with the speed. However, at the 0.5 -
TABLE II

Experiment: List of the Movement Speed and the Mean Stiffness

\begin{tabular}{c|c|c}
\hline $\begin{array}{c}\text { Voluntary Movement } \\
\text { Frequency }\end{array}$ & Naximum Speed & Meam Stifluess \\
Ilz & $\mathrm{deg} / \mathrm{s}(\mathrm{rad} / \mathrm{s})$ & $\mathrm{N}_{\mathrm{n}} / \mathrm{rad}$ \\
\hline 0.25 & $22.0(0.39)$ & 25.0 \\
0.5 & $90.0(1.5 \mathrm{~T})$ & 17.0 \\
1.0 & $180.0(3.14)$ & 32.0 \\
\hline
\end{tabular}

$\mathrm{Hz}$ voluntary movements, the stiffness mean value is twice as high as theirs. We think that their low stiffness mean value may be due to intertrial variation. More recently, Bennett [2] used the imposed constant position errors on the human elbow joint during voluntary movement to extract the dynamics. He found that the stiffness increased with the speed in the range of the maximum speed: 1-6 rad/s. However, the maximum stiffness from his experiment is below $15 \mathrm{Nm} / \mathrm{rad}$.

Regarding to the current movement theory, the stiffness increase with the speed in the speed range of $0.5-$ to $1-\mathrm{Hz}$ voluntary movement seems to support the speed scaling law [1]. However, the speed scaling law requires the stiffness to be proportional to the squared ratio of the speeds. From the 0.5 to $1-\mathrm{Hz}$ movement, the speed is doubled, and the stiffness is also doubled, not four times. Therefore, our results here only partially support the speed scaling law. Flash [4] showed in her simulation that in order to fit the hand trajectory well during normal movements, the joint stiffnesses must be equal to or higher than those in posture. Our result from $1-\mathrm{Hz}$ movement seems consistent with her simulation.

The validity of our results is supported by the simulation study, good agreement between the HMRED and EWLS methods, and the experimental control such that the conditions of the methods are met. The stiffness and damping variation from our movement experiments are not unexpected. It should be clear that no method will work for all conditions. What is important is that the limitations or conditions of a method be clearly identified and the experiment be controlled to meet the conditions.

Recently, researchers [8], [14], [22] have showed experimentally that the random position perturbation decreased the reflex response and the reflex gain in general is much smaller than intrinsic gain. However, the pulse position inputs can elicit very large reflex responses which indicates the reflex is position perturbation dependent. Our airjet actuator produces random force perturbations. Compared to the position perturbation, the force perturbation of about $4 N$ cannot cause the muscle into the saturation state and has far less possibilities to alter the intrinsic and reflex response of the joint [18]. The effect of the perturbation on the identification of the joint mechanical properties needs to be further researched on.

\section{REFERENCES}

[1] C. G. Atkeson and J. M. Hollerbach, "Kinematics features of unrestrained vertical arm movements," J. Neurosci., vol. 5, pp. 2318-2330, 1985. 
[2] D. J. Bennett, "Torques generated at the human elbow joint in response to constant position errors imposed during voluntary movements," Exp. Brian Res., vol. 88, pp. 433-442, 1992.

[3] D. J. Bennett, J. M. Hollerbach, I. W. Hunter, and Y. Xu, "Time-varying stiffness of human elbow joint during cyclic voluntary movement," Exp. Brian Res., vol. 95, pp. 488-498, 1993.

[4] T. Flash, "The control of hand equilibrium trajectories in multi-joint arm movement," Biol. Cybern., vol. 57, pp. 257-274, 1987.

[5] I. W. Hunter and R. E. Kearney, "Dynamics of human ankle stiffness: Variation with mean ankle torque," J. Biomech., vol. 15, pp. 747-752, 1982.

[6] R. E. Kearney and I. W. Hunter, "Dynamics of human ankle stiffness: Variation with displacement amplitude," J. Biomech., vol. 15, no. 753, pp. 753-756, 1982

[7] , "System identification of human joint dynamics," Crit. Rev. Biomed. Eng., vol. 18, no. 1, pp. 55-87, 1990.

[8] R. E. Kearney, R. B. Stein, and L. Parameswaran, "Identification of intrinsic and reflex contributions to human ankle stiffness dynamics," IEEE Trans. Biomed. Eng., vol. 44, June 1997.

[9] F. Lacquaniti, F. Licata, and J. F. Soechting, "The mechanical behavior of the human forearm in response to transient perturbations," Biol. Cybern., vol. 44, pp. 35-46, 1982.

[10] J. M. Lanman," "movement and the mechanical properties of the intact human elbow joint." Ph.D. dissertation, Dept. of Psych., MIT, Cambridge, MA, 1980.

[11] P. J. Lawrence and R. D. Dawson, "Identification of periodic nonstationary antenna stabilization control systems by cross correlation techniques," Proc. Inst. Elect. Eng., vol. 124, pp. 797-801, 1977.

[12] J. B. MacNeil, R. E. Kearney, and I. W. Hunter, "Identification of timevarying biological systems from ensemble data," IEEE Trans. Biomed. Eng., vol. 39, pp. 1213-1225, Dec. 1992.

[13] J. F. Soechting, J. R. Dufresne, and F. Lacquaniti, "Time-varying properties of myotatic response in man during some simple motor tasks," J. Neurophysiol., vol. 46, no. 6, pp. 1226-1243, Dec. 1981.

[14] R. B. Stein and R. E. Kearney, "Nonlinear behavior of stretch reflexes at the human ankle joint," J. Neurophysiol., vol. 73, no. 1, pp. 65-72, Jan. 1995.

[15] Y. Xu and J. M. Hollerbach, "Design and calibration of a $2 \mathrm{~d}$ airjet device for human motor control study," in Proc. 17th Annu. Conf. IEEE Eng. in Medicine and Biol. Soc., Sept. 1995.

[16] _ "Nonlinear time-varying identification of human arm joint mechanical properties using a portable pneumatic thruster," in Proc. 1997 American Control Conf., June 1997, pp. 3281-3285.

[17] _ "Single trial versus ensemble data methods for identification," in Proc. 15th Annu. Int. Conf. IEEE Eng. in Med. and Biol. Soc., Oct. 1993, pp. 1159-1160.

[18] _ , "Effect of force perturbation on elbow joint pulse response in posture," in Proc. 16th Annu. Conf. IEEE Eng. in Medicine and Biol. Soc., Nov. 1994, pp. 426-427.

[19] _ "Identification of human joint mechanical properties from single trial data," IEEE Trans. Biomed. Eng., to be published.

[20] Y. Xu, J. M. Hollerbach, and I. W. Hunter, "Time-varying identification of elbow joint dynamics using exponentially weighted least squares," in Proc. 13th Annu. Int. Conf. IEEE Eng. in Med. and Biol. Soc., Nov. 1991, pp. 2020-2021.

[21] Y. Xu, I. W. Hunter, J. M. Hollerbach, and D. J. Bennett, "An airject actuator system for identification of the human arm joint mechanical properties," IEEE Trans. Biomed. Eng., vol. 38, pp. 1111-1122, Nov. 1991.
[22] L. Q. Zhang and W. Z. Rymer, "Simultaneous and nonlinear identification of mechanical and reflex properties of human elbow joint muscles," IEEE Trans. Biomed. Eng., vol. 44, pp. 1192-1209, Dec. 1997.

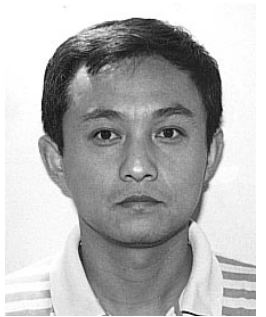

Yangming Xu (S'90-M'91) received the B.S. degree in mechanical engineering from Northwestern Polytechnical University, China, in 1982, and the M.S. and Ph.D. degrees in Aeronautics and Astronautics from Massachusetts Institute of Technology, Cambridge, in 1986 and 1991, respectively.

He jointed Sarcos Research Corporation (SRC) Utah, in 1994 as a Senior Control/Software Engineer working in the fields of real time control of robotic systems, whole body virtual interface, tactile display, and high-speed sensor network. He is also a Research Assistant Professor at Department of Mechanical Engineering, University of Utah, Salt Lake City. Before jointing SRC, he was a Postdoctoral Fellow in Biomedical Laboratory, at McGill University, Montreal, Quebec, Canada. His research interests include whole body interface to virtual world, human motor control, system identification, control of biological/robotic systems, and intelligent sensor network.

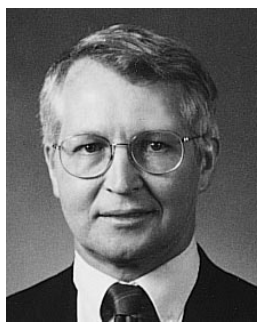

John M. Hollerbach (M'85-SM'92-F'96) received the B.S. degree in chemistry (1968) and the M.S degree in mathematics (1969) from the University of Michigan, and SM (1975) and Ph.D. (1978) degrees from Massachusetts Institute of Technology (MIT) in computer science.

$\mathrm{He}$ is Professor of Computer Science, and Research Professor of Bioengineering and of Mechanical Engineering, at the University of Utah, Salt Lake City. From 1989-1994 he was the Natural Sciences and Engineering/Canadian Institute for Advanced Research Professor of Robotics at McGill University, Montreal, P.Q., Canada, jointly in the Departments of Mechanical Engineering and Biomedical Engineering. From 1982-1989 he was on the faculty of the Department of Brain and Cognitive Sciences and a member of the Artificial Intelligence Laboratory at Massachusetts Institute of Technology (MIT), Cambridge: from 1978-1982 he was a Research Scientist.

In 1984, Dr. Hollerbach received a National Science Foundation (NSF) Presidential Young Investigator Award in 1988 and was named a Fellow of the Canadian Institute for Advanced Research. He was the Program Chairman of the 1989 IEEE International Conference on Robotics and Automation, a Member of the Administrative Committee of the IEEE Robotics and Automation Society from 1989-1993, Technical Editor of the IEEE TRANSACTIONS ON RoBOtiCS AND AUTOMATION from 1989-1994, and Treasurer of the IEEE/ASME Journal of Microelectromechanical Systems from 1992-1997. He was a member of the 1994-1995 National Research Council Committee on Virtual Reality Research and Development. Presently, he is a Senior Editor of Presence: Teleoperators and Virtual Environments, an Editorial Board member of the International Journal of Robotics Research, and a Governing Board member of the electronic journal Haptics-E. His research interests combine robotics, human motor control, teleoperation, virtual reality, and microelectromechanical systems. 